\title{
SUSTENTABILIDADE HÍDRICA URBANA: 0 CASO DE SANTA CATARINA
}

\author{
URBAN WATER SUSTAINABILITY: THE CASE OF SANTA CATARINA
}

Submissāo 26/03/2018

\section{Marcelo Pedroso Curtarelli Carlos Eduardo M. Tucci Vinicius Ragghianti Vitor Guimarães}

\section{RESUMO:}

A sustentabilidade hídrica e ambiental urbana é um dos grandes desafios da gestão de recursos hídricos. O ciclo hidrológico é fortemente alterado pela urbanização, produzindo impactos como inundações, contaminação do ar, do solo, rios e aquíferos, devido a forte concentração de população com grande demanda de água, energia, transporte e comunicaçáo e produzindo diferentes tipos de efluentes. Os serviços de água na cidade são o abastecimento de água, coleta e tratamento de esgoto, drenagem urbana e resíduos sólidos dentro de metas ambientais que busque a sustentabilidade do homem e do ambiente. O principal problema ambiental brasileiro no contexto urbano envolve o limitado serviço de coleta e tratamento de esgoto das cidades brasileiras. Tucci (2017) apresentou dois indicadores para avaliar a sustentabilidade urbana relacionada com o esgotamento sanitário, mostrando como estes limitados serviços influenciam o ambiente junto as cidades e sua área de influência. Neste artigo são apresentados os resultados destes indicadores para o estado de Santa Catarina, utilizados no diagnóstico do Plano Estadual de Recursos Hídricos de Santa Catarina. Os resultados mostram os indicadores em funçáo dos serviços atuais e apresentado um Plano de Investimentos para melhorar os serviços e os indicadores para os próximos 25 anos. Nos primeiros 15 anos foram previstos investimentos de 2,3\% do PIB resultando em reduçáo do indicador e dos impactos em $57 \%$. Na sequencia de uma fase de mais 5 anos com investimentos anuais da ordem de $\mathrm{R} \$ 63$ milhóes ( $0,02 \%$ do PIB) o indicador é reduzido para $60 \%$ com relação ao cenário atual e nos últimos cinco anos com $0,24 \%$ do PIB de investimento ao ano se obtém uma redução para $73 \%$ do Indicador no cenário atual.

Palavras chave: /sustentabilidade hídrica/ vazão/ esgoto sanitário/

\begin{abstract}
:
Water and environmental urban sustainability is one of the major challenges in water resources management. Hydrologic Cycle is impacted by urbanization, resulting in floods, air, soil, rivers and aquifers polluted due to high human concentration and great water, energy, transport and communication demand which results in many pollution sources. Water services in the cities are water supply, sanitation, stormwater, solids management with goals to achieve human and environment sustainability. The main environmental issue in Brazil in the urban areas is the lack of services on sanitation for Brazilian cities. Tucci (2017) presented two indicators to evaluate the urban sustainability related to sanitation, showing that these services impact the environment nearby the cities. This article presents the results of these indicators for the State of Santa Catarina using the Water Resource State Plan outputs. The results show the indicators in function of existing water and sanitation services and there were presented Plan of Investments in order to improve the services and indicators for the next 25 years.
\end{abstract}

Key-words: /water sustainability/flow/sewage/ 


\section{GESTÃO DE RECURSOS HÍDRICOS}

A gestáo de recursos hídricos disciplina o uso eficiente e sustentável da água (com base no instrumento da outorga), a qualidade da água dentro de padróes adequados (com o uso do instrumento de enquadramento) e o aumento da resiliência aos eventos extremos (visando a segurança hídrica). Estes aspectos envolvem os objetivos da legislação de recursos hídricos no Brasil (lei 9.433/1997) e de muitos países. Para atingir estes objetivos é necessária a construção de um gerenciamento adequado baseado na governança. Pahl-Wostl (2009) destacaram que o gerenciamento (management) envolvem as açóes para manter os limites de uso da água, da sua qualidade e segurança. A governança (governance) é a complexa relação de diferentes níveis de instituiçôes para tomada de decisão.

No Brasil este processo tem evoluído desde 1997, quando foi aprovada a legislação de recursos hídricos, mas tem apresentado algumas fragilidades relacionadas com a falta de sustentabilidade de governança, econômica, metodologia e ambiental. Os planos de recursos hídricos têm apresentado conteúdo de diagnóstico e pouco conteúdo sobre açóes efetivas com metas e investimentos, com indicadores de resultados, dentro dos três grandes grupos de açóes mencionados acima. Isto é resultado da falta de recursos financeiros, de indicadores apropriados para os objetivos da gestão da água do país e açóes definidas para a busca de atender metas de sustentabilidade hídrica.

\section{INDICADORES}

Os indicadores são utilizados para sintetizar o comportamento de um sistema e permitir sua avaliação. Um indicador pode ser definido como uma definição simplificada do mundo real (FEKETE; STAKHIV, 2014). Existem indicadores simples que permitem uma observação ou indicadores compostos que combinam vários outros indicadores.

Hooper (2006) propôs um conjunto de indicadores para gestão de recursos hídricos em bacias hidrográficas visando a aplicação na Gestão Integrada de Recursos Hídricos (GIRH). O autor selecionou 115 indicadores para organizaçóes de bacias dentro dos seguintes grupos: tomada de decisão coordenada; tomada de decisão relacionada com respostas; metas, mudança de meta e finalizaçáo de metas; sustentabilidade financeira; projeto de organização; legislação; treinamento; informação e pesquisa.

Fekete e Stakhiv (2014) mostram que existem indicadores de contexto, indicadores de funçáo, indicadores de governança e indicadores de resultados (performance). Os indicadores de contexto retratam, no caso da água, o contexto natural como a disponibilidade hídrica, precipitação, entre outros. Os indicadores de funçáo relacionam entrada e saída, como taxa de uso da água, tratamento de esgoto. Os indicadores de governança analisam diferentes níveis de eficiência, pelo uso de políticas, programas e regulaçôes. Os indicadores de eficiência ou resultado sintetizam o uso dos outros três indicadores para obter resultados para um setor específico, dentro de seus objetivos. Por exemplo, produtividade da água (water productivity) é a proporção do resultado econômico do uso da água em determinado setores.

\section{ÁGUAS URBANAS}

O desenvolvimento urbano necessita de água para abastecimento da populaçáo (abastecimento), de esgotamento da água utilizada, tratamento e disposição na natureza (esgotamento sanitário), da drenagem da água de chuva que precipita sobre a cidade e seu controle de quantidade e qualidade (drenagem urbana), gestáo dos sólidos, resultado dos sedimentos erodidos pela chuva e os resíduos produzidos pela população (resíduos sólidos). A gestão integrada destes serviços (águas urbanas) com o objetivo de atender a sustentabilidade da populaçáo e do ambiente é chamado de Gestáo Integrada de Águas Urbanas (GIAU) (TUCCI, 2009). O ciclo de contaminaçáo nas cidades é produzido quando o esgoto náo é tratado, a poluição da drenagem não é controlada, produzindo erosão e contaminação juntamente com os sólidos. Esta poluição contamina as fontes de água que perde disponibilidade hídrica por qualidade da água. Este é um problema de quase todas as cidades brasileiras. O principal fator deste problema tem sido a falta de tratamento de esgotamento sanitário. 


\section{Indicadores de abastecimento de água e esgotamento sanitário}

Os sistemas de abastecimento de água e esgotamento sanitário são fundamentais para atendimento da disponibilidade hídrica das áreas urbanas e o controle de seus efluentes. A realidade brasileira e de vários países em desenvolvimento tem sido de atendimento quase total para abastecimento de água e precário para esgotamento sanitário. Alguns dos indicadores destes serviços em nível brasileiro são sintetizados pelos indicadores da Tabela 1 .

Observam-se destes dados, que existe baixa eficiência no sistema de abastecimento com perdas (físicas e de faturamento) de 36,7\%. A vazão consuntiva de abastecimento de água é cerca de 20 a $25 \%$ da demanda, ou seja, 32,4 L/hab./dia (20\%). O restante retorna para a bacia (numa grande proporção poluída). O total de esgoto coletado e tratado é de $40,8 \%$ do total gerado, no entanto náo se conhece o nível de tratamento deste esgoto para os principais indicadores de poluição como o DBO (Demanda Bioquímica de Oxigênio). O SNIS que é o Sistema Nacional de Informaçôes em Saneamento não possui um indicador para avaliar o tratamento dos efluentes. Portanto, não é possível conhecer a efetiva carga ${ }^{1}$ que chega aos rios. Este seria o indicador que permitiria efetivamente conhecer a eficiência do sistema de tratamento de esgoto. $\mathrm{O}$ fato de existir coleta e tratamento, não garante que a carga foi eliminada ou reduzida, já que é significativo o número de Estações de Tratamento de Esgoto (ETE) funcionando de forma precária e com sistemas operacionais ineficientes.

Observa-se que o sistema de abastecimento de água e de esgoto são ineficientes e os indicadores usados não retratam a real situação dos mesmos no país. Analisando os dois sistemas de forma integrada, observa-se que a ineficiência no tratamento de esgoto faz com que uma parcela da vazão para abastecimento é reduzida devido a contaminação da fonte, ou seja, superficial ou subterrânea.

Para analisar a eficiência dos serviços de abastecimento de água e esgotamento sanitário foram identificados

1 Carga é o produto da vazão pela concentração do poluente, que pode ser obtido em unidades de $\mathrm{Kg} /$ dia ou Ton./dia. dois indicadores de resultado, chamados Vazão de subsidio $(Q)$ e Indice de sustentabilidade hidrica $\left(I S_{h}\right)$ (TUCCI, 2017).

Vazáo de subsídio - Tucci (2017) introduziu este conceito como sendo, a vazáo da natureza usada para recuperar a qualidade da água do rio, nos padróes de enquadramento do rio. O termo subsidio é usado porque a sociedade que náo faz a sua parte, coletando e tratando esgoto, está recebendo um subsídio da natureza. Portanto, neste conceito a demanda de água de uma área urbana não é somente a vazão consuntiva (consumida), mas deve ser acrescida da vazão necessária para diluir o esgoto náo tratado pela cidade. Para determinar esta vazáo foi adotado que esta vazão é obtida pela mistura da carga residual da cidade com a carga natural do rio. A carga residual depende do nível de coleta e tratamento de esgoto. A equação resultante é (TUCCI, 2017):

$Q_{s}=\frac{\propto . q\left[(1-\propto \beta) C_{n}-C_{s}\right]}{\left(C_{s}-C_{i}\right)}$

onde: $\mathrm{Q}_{\mathrm{s}}$ é a vazão de subsídio em L/dia; $\mathrm{C}_{\mathrm{s}}$ é a concentração meta da classe do rio para o parâmetro de qualidade água $(\mathrm{mg} / \mathrm{L}) ; \mathrm{C}_{\mathrm{i}}$ é a concentração do rio antes de receber o efluente, considerando esta uma concentração de rios com baixa poluição (menor ou igual a $\left.\mathrm{C}_{\mathrm{s}}\right)(\mathrm{mg} / \mathrm{L}) ; \mathrm{C}_{\mathrm{n}}$ é a concentração do parâmetro no esgoto bruto $(\mathrm{mg} / \mathrm{L})$; q é a vazáo da demanda de abastecimento da cidade em L/dia; $\propto$ é a parcela da demanda de água que retorna ao rio (varia entre 0 e 1 ), usualmente adotada igual a 0,$8 ; \beta$ é a parcela do esgoto que é coletada e tratada (varia entre 0 e 1 ); $\boldsymbol{\mu}$ é a proporção do esgoto bruto que é tratado (varia entre 0 e 1 ). Nesta expressão é possível introduzir o efeito das fossas de uma área urbana. Neste caso, a demanda total é reduzida da demanda que é distribuída para residências ou prédios comerciais e industriais que usam fossa.

Índice de Sustentabilidade Hídrica $\left(\right.$ IS $\left._{h}\right)$ - Este indicador é a relaçáo entre a demanda hídrica pela disponibilidade hídrica. No caso de uma cidade fica:

$I S_{h}=\frac{\text { demanda consuntiva }+ \text { vazão de subsídio }}{\text { disponibilidadehídrica }}$

Quando $\mathrm{IS}_{\mathrm{h}}>1$ significa que a demanda total é superior a disponibilidade e quando $\mathrm{IS}_{\mathrm{h}}<1$ indica que a demanda é inferior a disponibilidade. Geralmente 
Tabela 1 - Indicadores de abastecimento e saneamento do Brasil (SISTEMA NACIONAL DE INFORMAÇÓES SOBRE SANEAMENTO, 2015).

\begin{tabular}{llc}
\hline \multicolumn{1}{c}{ Setor } & \multicolumn{1}{c}{ Indicador } & Valor \\
\hline Abastecimento de água & Perdas na rede de distribuição (\%) & 36,7 \\
Abastecimento de água & Consumo médio por pessoa L/habitante/dia & 154 \\
Abastecimento e esgotamento sanitário & Proporção do volume consumido que deve ser tratado ${ }^{1}(\%)$ & 80 a 85 \\
Esgotamento sanitário & Cobertura de coleta e tratamento do total gerado (\%) & 40,8 \\
Esgotamento Sanitário & Nível de redução de DBO do esgoto bruto no tratamento (\%) & Desconhecido \\
\hline
\end{tabular}

${ }^{1}$ Observa-se que cerca de 15 a $20 \%$ do volume entregue pelo sistema de abastecimento é consumido no sistema.

quando na parte superior da equação é usada somente a demanda consuntiva observa-se uma grande folga nesta relação e $I_{h}$ é muito inferior a 1 . No entanto, quando a parte superior é somada a vazão de subsídio, raramente este indicador é inferior a 1 . Lembrando que este indicador representa um equilíbrio do abastecimento e das condiçóes ambientais da cidade e sua área próxima. Tucci (2017) apresentou a equação para este índice para uma área urbana área:

$I S_{h}=\frac{q \cdot p}{Q_{d}\left(C_{s}-C_{i}\right)}\left\{(1-\alpha)\left(C_{s}-C_{i}\right)+\alpha\left[(1-\mu \beta) C_{n}-C_{s}\right]\right\}$

onde: $\mathrm{p}$ = é a população em habitantes; $\mathrm{Q}_{\mathrm{d}}$ é a disponibilidade hídrica em L/dia.

Este índice pode ser utilizado para determinar o número de pessoas (para $\mathrm{IS}_{\mathrm{h}}=1$ ) que pode habitar uma área urbana dentro de determinados padróes de consumo de água $(\mathrm{q})$, coleta $(\beta)$ e tratamento de esgoto $(\mu)$ e disponibilidade hídrica $\mathrm{Q}_{\mathrm{d}}(\mathrm{L} / \mathrm{dia})$.

A disponibilidade hídrica pode ser definida pela disponibilidade da bacia hidrográfica a montante da cidade. Neste caso, a disponibilidade varia muito, de acordo com os mananciais disponíveis. Quando um rio contribui para a disponibilidade de várias cidades, na realidade a cidade mais a montante usa água limpa e as demais recebem água e esgoto, reduzindo a disponibilidade por falta de tratamento. Para caracterizar um limite da relação demanda-disponibilidade foi considerando a área de abrangência da cidade. Tucci (2017) converteu este índice para a relação entre a área de bacia necessária para atender a demanda dividida pela área de ocupação da cidade. Uma cidade sustentável deveria se limitar a usar a água que precipita na sua área de ocupação. Este conceito é discutível porque a maioria das cidades busca mananciais não urbanizados justamente por não controlar de forma sustentável seus impactos. A premissa é de que o índice determine quantas vezes a área urbana necessita de sua área de ocupação para atender a demanda total da cidade (incluindo a vazão de subsídio). Considerando que existe uma relação entre a disponibilidade hídrica e a área da bacia como a seguinte:

$Q_{d}=k \cdot q_{m} \cdot A$

onde: $\mathrm{Q}_{\mathrm{d}}$ é vazão de disponibilidade hídrica. Esta vazão, para uma bacia sem regularização, pode ser adotada igual a $Q_{95}$, (vazão de 95\% da curva de permanência, para rios sem regularização). No caso da existência de um reservatório, Q é vazão regularizada. Estas duas vazóes podem ser expressas em função da vazão média da bacia e esta, em função da área de drenagem; $\mathrm{k}$ é o coeficiente de regionalização entre $\mathrm{Q}_{95} / \mathrm{Q}_{\mathrm{m}}$ ou $\mathrm{Q}_{\mathrm{r}} / \mathrm{Q}_{\mathrm{m}}$ onde $\mathrm{Q}_{\mathrm{m}}$ é a vazão média em $\mathrm{L} /$ dia ou $\mathrm{m}^{3} / \mathrm{s}$. A vazão específica é $\mathrm{q}_{\mathrm{m}}=\mathrm{Q}_{\mathrm{m}} / \mathrm{A}$, onde $\mathrm{A}$ é a área de drenagem.

A população que ocupa a regiáo tem uma densidade de ocupação $\mathrm{D}=\mathrm{p} / \mathrm{A}_{\text {ocu }}$, onde $\mathrm{A}_{\text {ocu }}$ é a área de ocupação na mancha urbana. Substituindo esta equação e a equação 4 na equação 3 resulta na seguinte expressão para o indicador (Tucci, 2017):

$$
I S_{h}=\frac{\gamma}{f_{a}} \cdot\left\{(1-\alpha)\left(C_{s}-C_{i}\right)+\alpha\left[(1-\mu \beta) C_{n}-C_{s}\right]\right\}
$$

onde: $\gamma=\frac{q \cdot D}{86400 \cdot k \cdot q_{m}\left(C_{s}-C_{i}\right)}$. O coeficiente foi introduzido para considerar as unidades das variáveis; $\mathrm{f}_{\mathrm{a}}=\mathrm{A} / \mathrm{A}_{\text {ocu}}$. Quando $f_{a}>1$ significa que a cidade está usando uma área para disponibilidade hídrica superior a sua área de ocupação, devido a uma ou mais razóes: (a) a demanda consuntiva é muito alta; (b) a falta de tratamento de esgoto aumentou muito a vazão de subsídio. 
Para obter o equilíbrio é necessário atuar na eficiência do uso da água ou no tratamento dos efluentes.

\section{MATERIAIS E MÉTODOS}

\section{O ESTADO DE SANTA CATARINA}

Santa Catarina possuí um território de

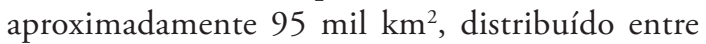
295 municípios e população urbana de aproximadamente 5,75 milhóes de habitantes (INSTITUTO BRASILEIRO
DE GEOGRAFIA E ESTATÍSTICA, 2017). A área urbanizada é de $1.700 \mathrm{~km}^{2}$ (GEOAMBIENTE SENSORIAMENTO REMOTO LTDA, 2008), representando apenas $1,79 \%$ da área do Estado. A densidade populacional urbana é de aproximadamente 34 hab./ha, inferior à densidade média brasileira de 65 hab./ha. O Estado é dividido em 10 Regióes Hidrográficas (RH) (ver Figura 1a), sendo 5 pertencentes a vertente do interior (RH1 a RH5) e 5 pertencentes a vertente do litoral (RH6 a RH10). A Figura 1 apresenta

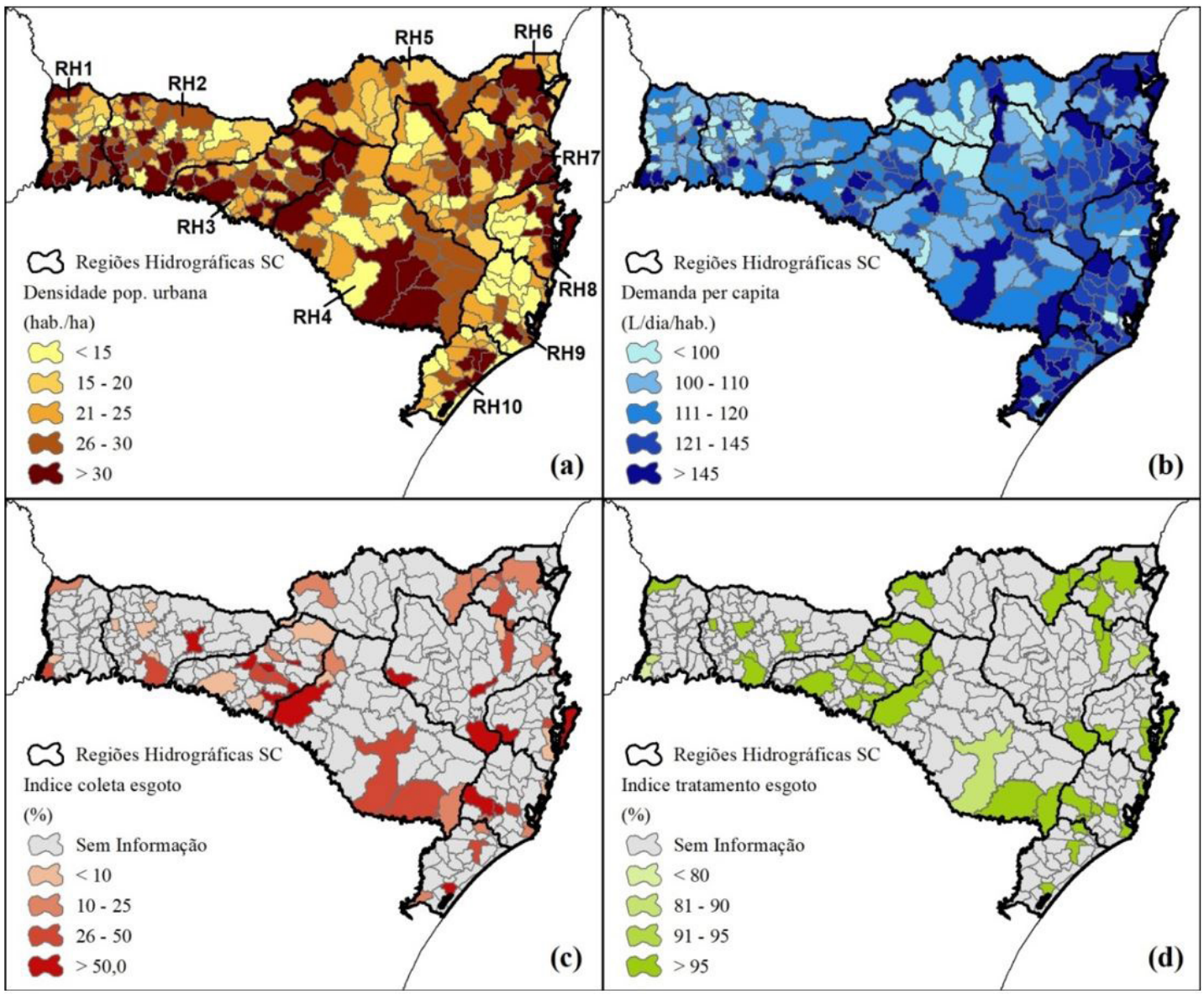

Figura 1. Área de estudo: (a) densidade populacional urbana (hab./ha - INSTITUTO BRASILEIRO DE GEOGRAFIA E ESTATÍSTICA, 2017; GEOAMBIENTE SENSORIAMENTO REMOTO LTDA, 2008), (b) consumo per capita (L/dia/hab. - SISTEMA NACIONAL DE INFORMAÇÓ́ES SOBRE SANEAMENTO, 2015), (c) índice de coleta de esgoto (\%, SISTEMA NACIONAL DE INFORMAÇÕES SOBRE SANEAMENTO, 2015) e (d) índice de tratamento de esgoto (\%, SISTEMA NACIONAL DE INFORMAÇÓEES SOBRE SANEAMENTO, 2015). 
a distribuição espacial da densidade populacional urbana e alguns indicadores de saneamento dos municípios catarinenses. Os principais indicadores de saneamento compilados para todo o Estado são apresentados na Tabela 2 .

\section{INDICADORES PARA O CENÁRIO ATUAL}

Para análise das condiçôes de qualidade da água dos rios foi adotado como indicador o DBO Demanda Bioquímica de Oxigênio, que é o principal indicador de carga de qualidade da água para fontes orgânicas. As variáveis a serem estimadas são: disponibilidade hídrica $Q_{d}$, demanda hídrica urbana $\mathrm{q}$, indicadores de coleta e tratamento de esgoto das cidades, meta de qualidade da água dos rios.

\section{Disponibilidade hídrica urbana}

A disponibilidade hídrica nas áreas urbanas dos municípios foi calculada com base no estudo de regionalização de vazão existente para Santa Catarina (2006). O referido estudo produziu equaçóes para as regiōes do Estado.

$$
Q_{d}=A^{a} \times P_{m}^{b} \times K \times C
$$

onde: $Q_{d}$ é o indicador de disponibilidade hídrica considerado (p.ex., $\mathrm{Q}_{90}, \mathrm{Q}_{95}, \mathrm{Q}_{98}$ ou outro) (L/dia), a, b e K são coeficientes da equação obtidos pelo referido estudo de regionalização; A é a área de drenagem considerada $\left(\mathrm{km}^{2}\right) ; \mathrm{P}_{\mathrm{m}}$ e a precipitação média anual na bacia ( $\mathrm{mm} / \mathrm{ano})$; $\mathrm{C}$ é uma constante utilizada para conversão de unidades $\left(=86,4 \times 10^{6}\right)$ - Comparando a Equação 4, k.q $q_{m}=K \cdot C \cdot P^{b} \cdot A^{a-1} \cdot A$ precipitação foi obtida através do Atlas Climatológico de Santa Catarina (PANDOLFO et al., 2002). A área de drenagem para computo da disponibilidade hídrica

Tabela 2 - Indicadores de saneamento de Santa Catarina (SISTEMA NACIONAL DE INFORMAÇÓES SOBRE SANEAMENTO, 2015).

\begin{tabular}{lc}
\hline \multicolumn{1}{c}{ Indicador } & Valor \\
\hline Demanda média per capita (L/dia/hab.) & 134 \\
Perdas na rede de distribuiçăo (\%) & 33 \\
Coleta de esgoto (\%) & 33 \\
Tratamento (\%) & 87 \\
\hline
\end{tabular}

foi considerada igual à área urbanizada/construída do município $\left(\mathrm{A}=\mathrm{A}_{\mathrm{ocu}}\right)$, obtida a partir do mapeamento de uso e ocupação do Estado de Santa Catarina (GEOAMBIENTE SENSORIAMENTO REMOTO LTDA, 2008). Foram utilizadas como indicadores de disponibilidade hídrica as vazóes com 95\% de permanência $\left(Q_{95}\right)$ e $98 \%$ de permanência $\left(Q_{98}\right)$. Estas vazóes correspondem a disponibilidade hídrica sem regularização com um risco 5\% e 2\% de falha.

\section{Demanda hídrica urbana}

O cálculo da demanda hídrica urbana dos municípios de Santa Catarina foi realizado com base nas informaçóes populacionais disponibilizadas pelo Instituto Brasileiro de Geografia e Estatística (IBGE) e informações de saneamento SNIS. Neste caso a demanda hídrica urbana total de um dado município, $\mathrm{Q}_{\mathrm{T}}$ (L/dia), foi obtida como na equação 2 pela seguinte equação ${ }^{2}$ :

$$
Q_{T}=Q_{c}+Q_{s}
$$

onde: o termo $\mathrm{Q}_{\mathrm{c}}$ representa o consumo direto da água nas residências e prédios enquanto o termo $\mathrm{Q}_{\mathrm{s}}$ representa a vazão de subsídio que os corpos d'água tem que prover para diluir os efluentes lançados e mantê-los em níveis de qualidade aceitáveis, conforme preconizado na Resolução CONAMA 357/05 (Equaçáo 1). O consumo direto de água nas residências, foi calculado utilizando a seguinte equação:

$$
Q_{c}=q_{p c} \times p \times(1-\alpha)
$$

onde: $\mathrm{q}_{\mathrm{pc}}$ é o consumo per capita do município (L/dia/hab.), conforme informaçôes do Sistema Nacional de Informaçôes sobre Saneamento (2015), p é a população urbana do município (habitantes), conforme Instituto Brasileiro de Geografia e Estatística (2017), $\alpha$ é um coeficiente de retorno (adimensional), considerado 0,8 .

A vazão de subsídio, $Q_{S}$ (L/dia), foi calculada utilizando a Equação 1. Os valores de $\beta$ para cada município foram obtidos utilizando os índices de coleta

2 Neste trabalho não foi considerada a parcela de consumo relativa a "água virtual", isto é, a quantidade de água usada nos produtos que usamos ou consumimos no nosso dia a dia. 
e tratamento disponibilizados no SNIS (SISTEMA NACIONAL DE INFORMAÇÓES SOBRE SANEAMENTO, 2015) (IN015_AE e IN016_AE), complementados com dados do Instituto Brasileiro de Geografia e Estatística (2010). Devido a falta de dados sobre o nível (eficiência) do tratamento $(\mu)$, foi adotado para todos os municípios um valor de $80 \%$. $\mathrm{O}$ parâmetro de qualidade da água utilizado para avaliar a $Q_{s}$ foi a Demanda Biológica de Oxigênio (DBO). Neste caso $\mathrm{C}_{\mathrm{n}}$ foi considerada igual a $300 \mathrm{mg} / \mathrm{l}$ (VON SPERLING, 2005), $\mathrm{C}_{\mathrm{s}}=5 \mathrm{mg} / \mathrm{l}$ para rio classe 2 (BRASIL, 2005) e $\mathrm{C}_{\mathrm{i}}=2 \mathrm{mg} / \mathrm{l}$ para condiçóes naturais esperadas.

\section{Indicador de sustentabilidade hídrica urbana}

O Indicador de Sustentabilidade Hídrica Urbana $\left(\right.$ IS $_{\mathrm{h}}$ ) (TUCCI, 2017), relaciona a demanda total de água no ambiente urbano, $\mathrm{Q}_{\mathrm{T}}$, e a disponibilidade hídrica possível de ser gerada no ambiente urbano, $Q_{d}$. Este índice foi calculado considerando a proporção das áreas de disponibilidade hídrica e de ocupação (Equação 5)

Os resultados obtidos para o $\mathrm{IS}_{\mathrm{h}}$ em nível de municípios foram agregados para o nível de Região Hidrográfica $(\mathrm{RH})$ levando em conta a posição das áreas urbanas e os limites das RH. A abrangência municipal e localização das sedes urbanas em relação as RH foram obtidas através dos relatórios de caracterização geral elaborados no âmbito do Plano Estadual de Recursos Hídricos de Santa Catarina (PERH/SC) ${ }^{3}$.

\section{RESULTADOS}

Os resultados apresentados a seguir mostram: (a) o diagnóstico da situação atual quanto a vazão de subsídio e o índice de sustentabilidade; (b) os cenários de intervençóes potenciais considerando um programa de investimentos de 15 anos, com base numa proporção PIB estadual em Saneamento.

\section{Diagnóstico}

A Tabela 3 apresenta um resumo dos valores de uso da água e coleta e tratamento de esgoto pela

3 Disponibilizado em http://www.aguas.sc.gov.br/ base-documental/plano-estadulal-biblioteca.
RH do Estado. A maior parte da população vive nas RH litorâneas (RH6, RH7, RH8, RH9 e RH10), aproximadamente $73 \%$ do total do Estado. Neste aspecto, destacam-se as RH7 (Vale do Itajaí) e RH8 (Litoral Centro), onde vivem $44 \%$ da população e estáo localizadas 7 das 12 cidades com mais de 100 mil habitantes no Estado. Ainda com relação a aspectos demográficos, a densidade populacional urbana ultrapassa os 100 hab./ha na RH8, indicando alto grau de verticalização nas cidades desta RH.

A demanda per capita para abastecimento urbano nas RH varia ente 111 (RH2) e 166 (RH7) L/dia/hab., o índice de coleta e tratamento de esgoto varia entre $10 \%$ (RH1) e 49\% (RH4), sendo os maiores valores observados nas RH litorâneas (com exceção da RH4 - Planalto de Lages). A carga total gerada pelo Estado nas áreas urbanas é de 200,8 mil ton/dia de $\mathrm{DBO}$ e deste total $54,8 \mathrm{mil}$ ton/dia é tratado e $146 \mathrm{mil}$ ton/dia de DBO despejado nos rios sem tratamento, representando uma carga retirada de apenas 37,5\%.

A Figura 2 apresenta a distribuição espacial dos termos que compóe a demanda total de água no meio urbano considerando o cenário atual dos municípios catarinenses. Com relaçáo ao termo do consumo direto, $\mathrm{Q}_{\mathrm{c}}$, (Figura 2a), os valores variaram entre $0,0001 \mathrm{~m}^{3} / \mathrm{s}$ (em Tigrinhos) e $0,19 \mathrm{~m}^{3} / \mathrm{s}$ (em Joinville), com valor médio para os municípios do Estado de $0,007 \mathrm{~m}^{3} / \mathrm{s}$. Considerando o somatório do consumo direto de todos os municípios, o valor global para Santa Catarina é de aproximadamente $1,97 \mathrm{~m}^{3} / \mathrm{s}\left(\sim 0,03 \mathrm{~m}^{3} / \mathrm{dia} / \mathrm{hab}\right.$. $)$. A vazão de subsídio, por sua vez, variou entre $0,03 \mathrm{~m}^{3} / \mathrm{s}$ (em Tigrinhos) e $55 \mathrm{~m}^{3} / \mathrm{s}$ (em Joinville), com valor médio para os municípios do Estado de $1,9 \mathrm{~m}^{3} / \mathrm{s}$. Considerando o somatório de todos os municípios, o valor global para Santa Catarina é de aproximadamente $554 \mathrm{~m}^{3} / \mathrm{s}\left(-8,50 \mathrm{~m}^{3} / \mathrm{dia} / \mathrm{hab}\right.$.). A demanda total é de $556 \mathrm{~m}^{3} / \mathrm{s}\left(8,53 \mathrm{~m}^{3} / \mathrm{dia} / \mathrm{hab}\right.$.), portanto a vazão de subsídio representa 99,6\% da vazão total. No Brasil, o consumo direto no meio urbano é de $76,39 \mathrm{~m}^{3} / \mathrm{s}$ $\left(0,033 \mathrm{~m}^{3} / \mathrm{dia} / \mathrm{hab}\right.$.). A vazão de subsídio $\mathrm{Q}_{\mathrm{S}}$ é cerca de $17.360 \mathrm{de} \mathrm{m}^{3} / \mathrm{s}\left(7,50 \mathrm{~m}^{3} / \mathrm{dia} / \mathrm{hab}.\right)$, resultando em uma demanda total de $7,53 \mathrm{~m}^{3} /$ hab./dia. Assim, constata-se que a demanda total per capita obtida para as cidades de Santa Catarina é 13\% superior ao verificado para o Brasil como um todo. 
Tabela 3 - Resumo do diagnóstico por RH de Santa Catarina.

\begin{tabular}{cccccccc}
\hline RH & $\begin{array}{c}\text { Populaçáo } \\
\text { Urbana } \\
\text { (hab.) }\end{array}$ & $\begin{array}{c}\text { Densidade } \\
\text { populacional } \\
\text { urbana } \\
\text { (hab./ha) }\end{array}$ & $\begin{array}{c}\text { Demanda per } \\
\text { capita } \\
\text { (L/dia/hab.) }\end{array}$ & $\begin{array}{c}\text { Coleta e } \\
\text { tratamento de } \\
\text { esgoto } \\
(\%)\end{array}$ & $\begin{array}{c}\text { Carga total } \\
(1000 \text { ton./dia } \\
\text { de DBO) }\end{array}$ & $\begin{array}{c}\text { Carga tratada } \\
(1000 \text { ton./dia } \\
\text { de DBO) }\end{array}$ & $\begin{array}{c}\text { Carga para os } \\
\text { rios } \\
(1000 \text { ton./dia } \\
\text { de DBO) }\end{array}$ \\
\hline 1 & 160.394 & 34 & 117 & 10 & 4,5 & 0,36 & 4,14 \\
2 & 381.340 & 39 & 111 & 18 & 10,2 & 1,46 & 8,70 \\
3 & 339.640 & 43 & 122 & 24 & 9,9 & 1,91 & 8,04 \\
4 & 394.723 & 45 & 121 & 49 & 11,5 & 4,49 & 6,97 \\
5 & 299.119 & 37 & 117 & 14 & 8,4 & 0,94 & 7,46 \\
6 & 869.513 & 43 & 160 & 37 & 33,4 & 9,88 & 23,51 \\
7 & 1.318 .576 & 47 & 166 & 32 & 52,5 & 13,45 & 39,08 \\
8 & 1.191 .356 & 126 & 155 & 39 & 44,3 & 13,83 & 30,49 \\
9 & 314.901 & 47 & 137 & 35 & 10,4 & 2,90 & 7,45 \\
10 & 478.860 & 37 & 137 & 44 & 15,7 & 5,54 & 10,20 \\
Total & $\mathbf{5 . 7 4 8 . 4 2 2}$ & $\mathbf{3 4}$ & $\mathbf{1 3 4}$ & $\mathbf{2 9}$ & $\mathbf{2 0 0 , 8}$ & $\mathbf{5 4 , 8}$ & $\mathbf{1 4 6 , 0}$ \\
\hline
\end{tabular}

* Destacado em vermelho estão as RH localizadas na vertente do interior e em azul as RH localizadas na vertente litorânea.

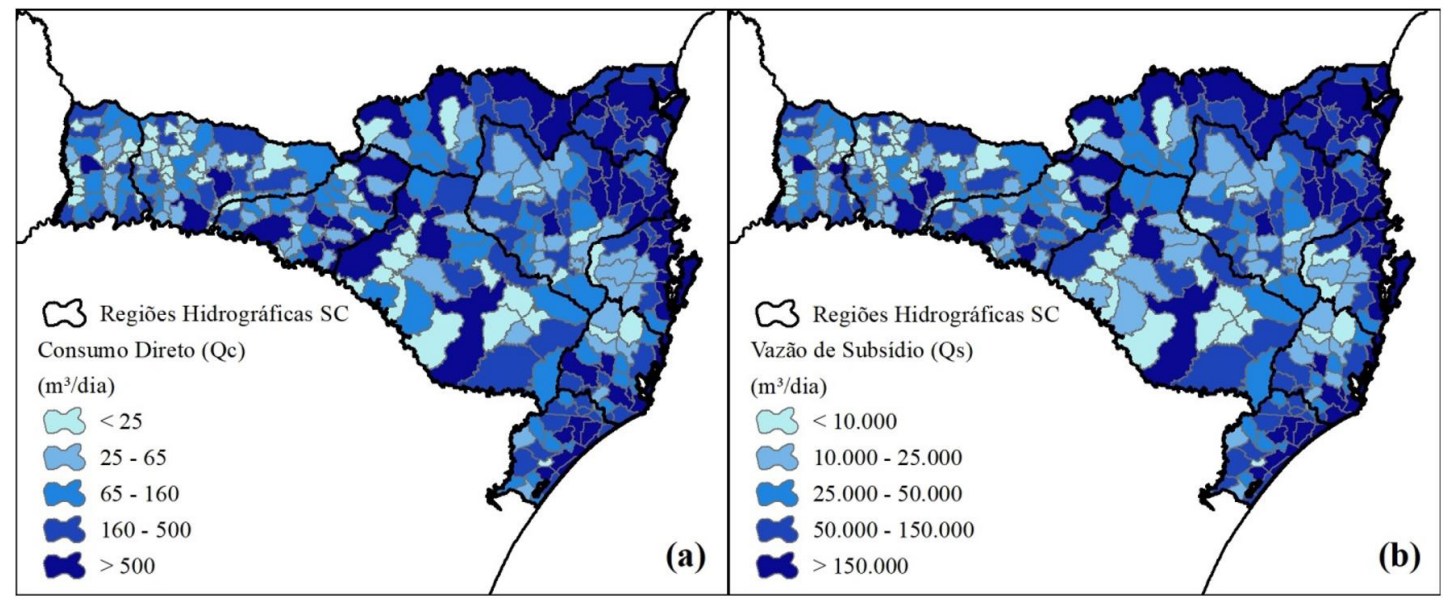

Figura 2. Estimativa da demanda total de água no meio urbano: (a) consumo direto $\left(\mathrm{m}^{3} / \mathrm{dia}\right)$ e (b) vazão de subsídio para diluição de cargas orgânicas domésticas $\left(\mathrm{m}^{3} / \mathrm{dia}\right)$.

A análise conjunta dos dois termos considerados no cálculo da demanda total de água $\left(\mathrm{Q}_{C}\right.$ e $\left.\mathrm{Q}_{S}\right)$ mostra que, na média, nos municípios catarinenses a parcela da demanda total relacionada ao termo $\mathrm{Q}_{S}$ é cerca de 280 vezes superior à relacionada ao termo $Q_{C}$. Estes valores indicam que a demanda direta é insignificante perto da demanda para recuperar os recursos hídricos da contaminação pelos efluentes domésticos urbanos, representada pelo termo $\mathrm{Q}_{S}$. Estes resultados revelam a magnitude do impacto da falta de saneamento no meio urbano, que reduzem drasticamente a disponibilidade hídrica por falta de qualidade da água.

A Figura 3 apresenta o $\mathrm{IS}_{\mathrm{h}}$ calculado para os 295 municípios de Santa Catarina levando em conta diferentes indicadores de disponibilidade hídrica considerados neste estudo. Como pode ser observado, tanto considerando a $\mathrm{Q}_{95}$ (Figura 3a) como a $\mathrm{Q}_{98}$ (Figura 3b) como indicador de disponibilidade hídrica, 


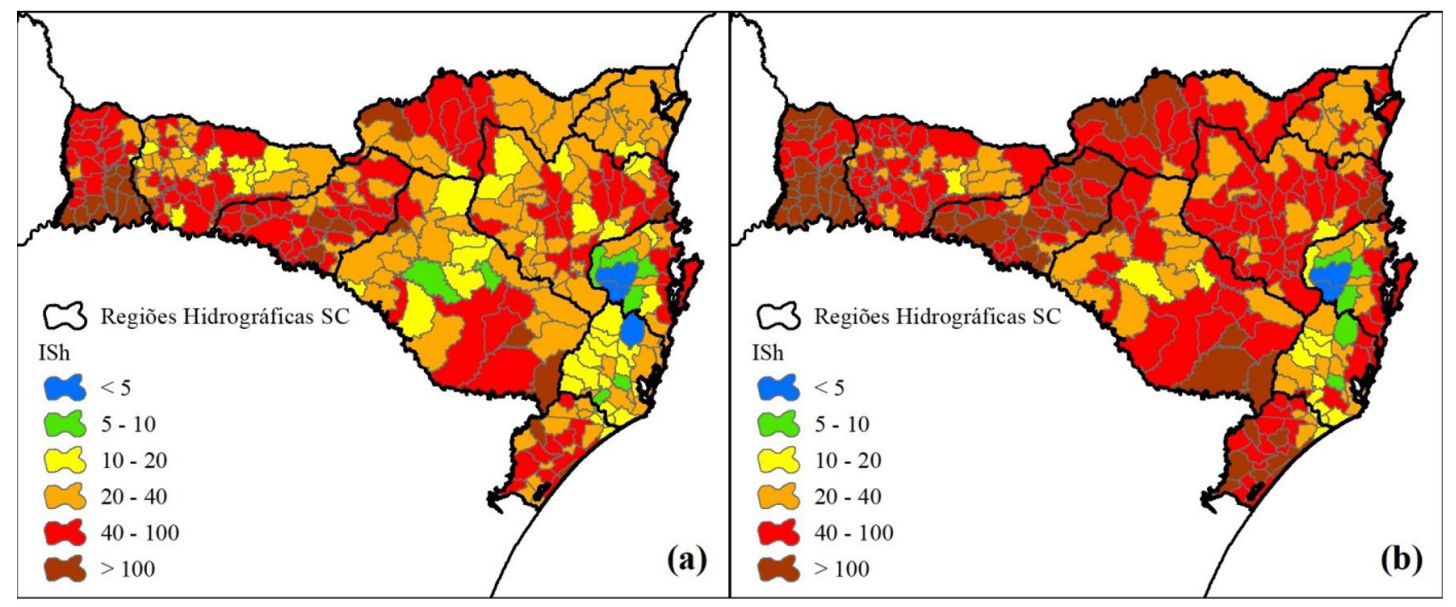

Figura 3. Indicador de sustentabilidade hídrica no ambiente urbano: (a) indicador de disponibilidade hídrica $\mathrm{Q}_{95}$ e (b) indicador de disponibilidade hídrica $\mathrm{Q}_{98}$.

todos os 295 municípios de Santa Catarina apresentam IS $_{\mathrm{h}}$ superior a 1, ou seja, condiçóes insustentáveis com relação a gestão urbana dos recursos hídricos.

Levando em conta a $\mathrm{Q}_{95}$ como indicador de disponibilidade hídrica (Figura 3a), os valores do $\mathrm{IS}_{\mathrm{h}}$ variaram entre 1,87 (em rancho Queimado - melhor desempenho) e 234,1 (em Mondaí - pior desempenho), com valor global para o Estado de 44,12. Por sua vez, considerando a $\mathrm{Q}_{98}$ como o indicador de disponibilidade hídrica (Figura $3 \mathrm{~b}$ ), os valores do $\mathrm{IS}_{\mathrm{h}}$ variaram entre 2,27 (em Rancho Queimado - melhor desempenho) e 367,88 (em Mondaí - melhor desempenho), com valor global para o Santa Catarina de 58,59.

A análise dos valores globais de IS $_{h}$ evidenciam a insustentabilidade hídrica no meio urbano de Santa Catarina. Este cenário de insustentabilidade fica evidente levando em conta que as cidades catarinenses apresentam, na média, uma demanda total de água entre 44 a 59 vezes a disponibilidade obtida em uma área igual a sua área urbanizada. Neste contexto, para que as cidades catarinenses se tornem sustentáveis do ponto de vista da gestão hídrica algumas soluçóes podem ser apontadas: (1) ampliar a capacidade de prover água, ou seja, a disponibilidade hídrica no meio urbano; e (2) ampliar e melhorar o desempenho dos sistemas de saneamento.

A partir dos resultados obtidos por município, os valores de consumo direto, vazão de subsídio, demanda hídrica urbana total, e indicadores de sustentabilidade hídrica foram agregados para o nível de $\mathrm{RH}$, sendo os resultados apresentados na Tabela 4. Para o cenário atual, a demanda hídrica urbana de total per capita variou entre 5,9 m²/dia (RH4) e 9,4 (RH7) $\mathrm{m}^{3} / \mathrm{dia}$. Considerando os valores totais por região a demanda hídrica urbana varia entre 15,1 (RH1) e 144 (RH7) $\mathrm{m}^{3} / \mathrm{s}$. Com relação ao índice de sustentabilidade hídrica, o melhor desempenho é observado na RH9, enquanto na RH1 é observado o pior desempenho.

\section{Plano de Investimento}

A elaboração do cenário de investimento necessário para melhorar o desempenho das cidades catarinenses em relação à sustentabilidade hídrica considerou três fases distintas de implementação e levou em conta apenas aspectos relacionados à ampliaçáo da coleta e tratamento de esgoto e eficiência na remoção da carga poluente. Assim não foram considerados aspectos relacionados à ampliação da disponibilidade hídrica nas cidades do Estado. O aumento de disponibilidade hídrica somente poderia ser obtido com a regularização de vazão ${ }^{4}$. Os cenários são os seguintes:

4 Usar a regularização para aumentar a sustentabilidade a qualidade da água seria tratar esgoto com regularização, o que é ambientalmente inadequado; 
Tabela 4 - Cenário atual da demanda hídrica urbana e índice de sustentabilidade hídrica por RH.

\begin{tabular}{ccccccc}
\hline $\mathrm{RH}$ & $\mathrm{Q}_{\mathrm{C}}\left(\mathrm{m}^{3} / \mathrm{s}\right)$ & $\mathrm{Q}_{\mathrm{S}}\left(\mathrm{m}^{3} / \mathrm{s}\right)$ & $\mathrm{Q}_{\mathrm{T}}\left(\mathrm{m}^{3} / \mathrm{s}\right)$ & $\begin{array}{c}\mathrm{Q}_{\mathrm{T}} \text { per capita } \\
\left(\mathrm{m}^{3} / \text { dia/hab. }\right)\end{array}$ & $\mathrm{IS}_{\mathrm{h}}\left(\mathrm{Q}_{95}\right)$ & $\mathrm{IS}_{\mathrm{h}}\left(\mathrm{Q}_{98}\right)$ \\
\hline 1 & 0,04 & 15,10 & 15,14 & 8,16 & 103 & 162 \\
2 & 0,10 & 33,24 & 33,34 & 7,55 & 50 & 69 \\
3 & 0,10 & 30,39 & 30,48 & 7,75 & 76 & 110 \\
4 & 0,12 & 26,87 & 26,99 & 5,91 & 39 & 55 \\
5 & 0,08 & 27,89 & 27,97 & 8,08 & 42 & 54 \\
6 & 0,32 & 90,82 & 91,14 & 9,06 & 29 & 37 \\
7 & 0,50 & 143,08 & 143,59 & 9,41 & 58 & 82 \\
8 & 0,44 & 114,41 & 114,84 & 8,33 & 45 & 55 \\
9 & 0,10 & 27,49 & 27,59 & 7,57 & 23 & 30 \\
10 & 0,17 & 44,84 & 45,00 & 8,12 & 51 & 78 \\
TOTAL & $\mathbf{1 , 9 7}$ & $\mathbf{5 5 4 , 1 2}$ & $\mathbf{5 5 6 , 0 8}$ & $\mathbf{7 , 9 9 *}$ & $\mathbf{4 3 , 4 5 *}$ & $\mathbf{5 7 , 7 *}$ \\
\hline
\end{tabular}

* Valores médios.

Fase A - A meta adotada foi de $95 \%$ da população urbana de Santa Catarina atendida com coleta e tratamento de esgoto com uma eficiência de remoção da carga poluente de $80 \%$. Os investimentos foram escalonados em um horizonte de 15 anos, partindo das condiçóes atuais de esgotamento sanitário nas cidades catarinenses. Neste caso, foram considerados incrementos regulares ao ano no índice de coleta $\mathrm{e}$ tratamento, partindo da situação atual de cada município até os $95 \%$ finais no ano 15 . Foi considerado um custo médio de R \$ 1.400 por habitante para implantação da infraestrutura de coleta e tratamento de esgoto (AGÊNCIA NACIONAL DE ÁGUAS, 2017).

Fase B - A meta final 98\% da população urbana com acesso a coleta de e tratamento de esgoto e $80 \%$ de eficiência na remoção da carga poluente. $\mathrm{O}$ horizonte de tempo considerado para atingir a meta foi de 5 anos, após o fase $\mathrm{A}$.

Fase C - Meta final 100\% de esgoto coletado e tratado nas 12 cidades de Santa Catarina com mais de 100 mil habitantes e eficiência de $95 \%$ na remoção da carga poluente nestes municípios. O horizonte de tempo considerado para atingir a meta também foi de 5 anos, após a fase $B$.

Para as 3 fases de investimento simuladas foi considerada a taxa de crescimento anual da população urbana, calculada de acordo com os dados do censo IBGE para os municípios catarinenses (INSTITUTO BRASILEIRO DE GEOGRAFIA E ESTATÍSTICA,
2017). Neste caso, o crescimento médio da população urbana de Santa Catarina foi considerado igual a 1,5\% ao ano, sendo esta taxa considerada constante ao longo dos horizontes de investimento. Provavelmente esta taxa será revista, já que a fertilidade no Sul do Brasil não sustenta esta taxa de crescimento.

A Figura 4 apresenta os resultados da simulação dos investimentos necessários, considerando os três cenários e metas descritas acima. Os investimentos necessários para alcançar a meta da fase A são de aproximadamente $\mathrm{R} \$ 5,6$ bilhóes, valor que representa 2,23\% do Produto Interno Bruto (PIB) atual do Estado ${ }^{5}$. Considerando que os investimentos desta fase serão escalonados em 15 anos, o custo médio anual é de aproximadamente $\mathrm{R} \$ 371$ milhóes, pouco mais de $0,12 \%$ do PIB. Como resultado deste investimento, espera-se ao fim da fase A uma redução de $66 \%$ na vazão de subsídio per capita no estado, indo dos atuais $8,33 \mathrm{~m}^{3} / \mathrm{dia} / \mathrm{hab}$. para $2,81 \mathrm{~m}^{3} / \mathrm{dia} / \mathrm{hab}$.. Com relação ao indicador de sustentabilidade hídrica, ao final da fase A espera-se uma redução de $58 \%$ em comparação ao cenário atual.

Para o cumprimento da meta da fase B foi estimado um investimento adicional de $\mathrm{R} \$ 313$ milhóes aplicados em 5 anos, o que representa na média $\mathrm{R} \$ 63$ milhóes investidos a cada ano. Como resultado desta fase de investimento, espera-se uma redução de 9,8\% na vazão

5 R\$ 250 bilhôes no ano 2015. 


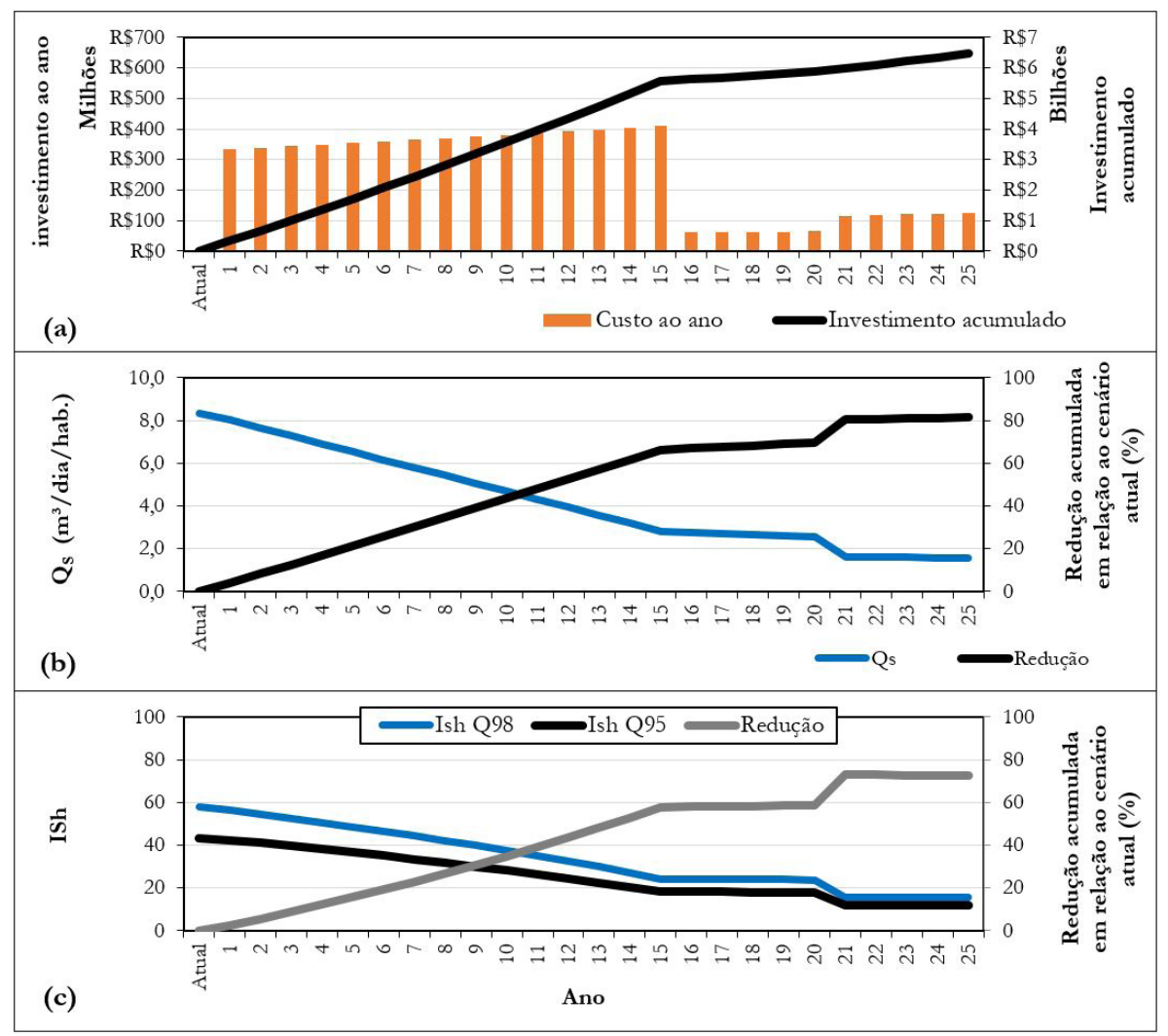

Figura 4. Evolução ao longo dos horizontes de investimentos: (a) investimentos ao ano e acumulado, (b) reflexos na vazão de subsídio (Qs), e (c) evolução do indicador de sustentabilidade hídrica.

de subsídio per capita em comparação ao resultado obtido ao final da fase $\mathrm{A}$, indo de $2,81 \mathrm{~m}^{3} / \mathrm{dia} / \mathrm{hab}$. para $2,54 \mathrm{~m}^{3} / \mathrm{dia} / \mathrm{hab}$.. Para o indicador de sustentabilidade hídrica, espera-se uma redução de aproximadamente $3 \%$ em relação ao resultado obtido ao final da fase A.

Por fim, para a fase $\mathrm{C}$ foi estimado um investimento de R\$ 602 milhóes em 5 anos, o que representa, na média, R\$ 120 milhóes investidos a cada ano. Espera-se ao final da fase $\mathrm{C}$ uma redução de aproximadamente $39 \%$ na vazão de subsídio per capita em comparação aos resultados obtidos ao final da fase $\mathrm{B}$, indo de $2,54 \mathrm{~m}^{3} / \mathrm{dia} / \mathrm{hab}$. para $1,55 \mathrm{~m}^{3} / \mathrm{dia} / \mathrm{hab}$.. Para o indicador de sustentabilidade hídrica é esperado uma redução de 33\% em comparaçáo ao resultado obtido ao final da fase B.
$\mathrm{O}$ valor global de investimentos considerando as fases A, B e C é de R \$ 6,5 bilhóes, equivalente a 2,6\% do PIB atual de Santa Catarina. Espera-se ao final das três fases uma redução de $81 \%$ na vazão de subsídio per capita do Estado, indo de $8,33 \mathrm{~m}^{3} / \mathrm{dia} / \mathrm{hab}$. para $1,55 \mathrm{~m}^{3} / \mathrm{dia} /$ hab.. Para o indicador de sustentabilidade hídrica espera-se uma redução de $72 \%$ em comparação ao cenário atual.

A Tabela 5 apresenta os valores de investimento em cada uma das fases simuladas e o respectivo impacto sobre o $\mathrm{IS}_{\mathrm{h}}$, compilados para cada uma das RH do Estado de Santa Catarina. Como esperado, os maiores valores de investimentos devem ser alocados nas RH7, RH8 e RH6, as mais populosas do Estado e que apesentam maiores valores de carga lançada em corpos d'água no cenário atual. $\mathrm{O}$ principal impacto 
dos investimentos sobre o IS $\mathrm{h}$ é verificado na RH6, onde espera-se que ocorra uma redução de $80 \%$ do valor em relação ao cenário atual. Por sua vez, o menor impacto \% é esperado na RH9, onde se espera que ao final das 3 fases de investimento ocorra uma redução de $56 \%$ do $\mathrm{IS}_{\mathrm{h}}$ em relação ao cenário atual. A evolução do IS $_{\mathrm{h}}$ ao final de cada uma das fases de investimento e para cada RH são apresentados na Figura 5.

Tabela 5 - Resumo dos cenários de investimento por RH e o respectivo impacto sobre o índice de sustentabilidade hídrica.

\begin{tabular}{|c|c|c|c|c|c|c|c|c|}
\hline \multirow[b]{2}{*}{$\mathrm{RH}$} & \multicolumn{4}{|c|}{ Investimento (milhões de R\$) } & \multicolumn{4}{|c|}{ Redução do IS $(\%)$} \\
\hline & Fase A & Fase B & Fase C & Total & $\begin{array}{c}\text { Atual - } \\
\text { Fase A }\end{array}$ & $\begin{array}{c}\text { Fase A - } \\
\text { Fase B }\end{array}$ & $\begin{array}{c}\text { Fase B - } \\
\text { Fase C }\end{array}$ & Total \\
\hline 1 & 206 & 8 & 21 & 236 & 66 & 2,90 & 0,00 & 64,22 \\
\hline 2 & 472 & 22 & 54 & 547 & 64 & 0,93 & 26,39 & 73,49 \\
\hline 3 & 359 & 17 & 33 & 409 & 64 & 4,83 & 0,00 & 64,12 \\
\hline 4 & 239 & 17 & 19 & 275 & 56 & 7,89 & 29,36 & 71,42 \\
\hline 5 & 347 & 14 & 17 & 377 & 69 & 6,74 & 0,00 & 70,42 \\
\hline 6 & 845 & 50 & 73 & 967 & 58 & 2,82 & 51,81 & 80,24 \\
\hline 7 & 1.425 & 79 & 165 & 1.669 & 56 & 1,29 & 39,13 & 73,71 \\
\hline 8 & 988 & 64 & 130 & 1.183 & 54 & 1,75 & 46,61 & 75,69 \\
\hline 9 & 286 & 17 & 41 & 344 & 57 & 3,39 & 0,00 & 55,89 \\
\hline 10 & 396 & 26 & 50 & 472 & 56 & 2,89 & 21,42 & 66,47 \\
\hline TOTAL & 5.563 & 314 & 602 & 6.479 & $60^{*}$ & $3,5^{*}$ & $21,5^{*}$ & $69,6^{*}$ \\
\hline
\end{tabular}

* Valores médios.

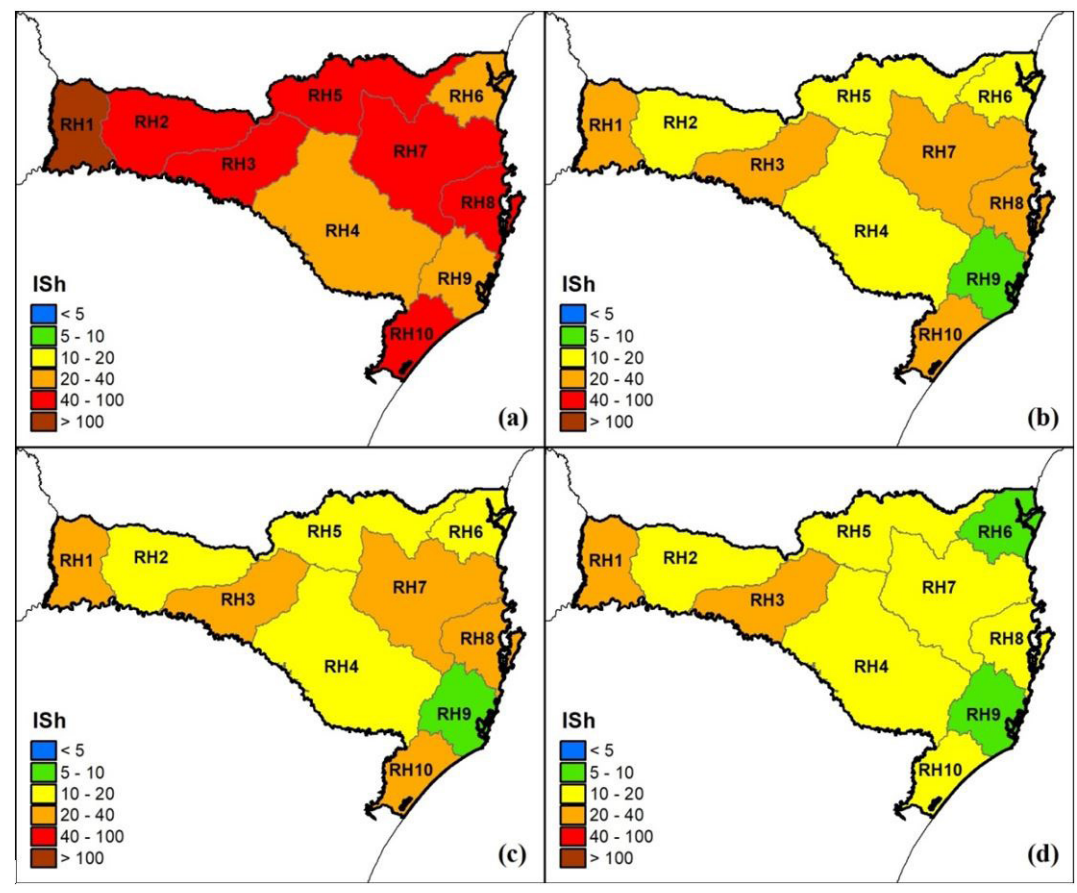

Figura 5. Evolução do ISh por RH de Santa Catarina: (a) Cenário atual; (b) ISh ao final da fase A de investimento; (c) ISh ao final da fase B de investimento; (d) ISh ao final da fase C de investimento. 


\section{CONCLUSÓES}

Atualmente o saneamento tem utilizado indicadores de abastecimento de água e tratamento de esgoto que não retratam a realidade das condiçóes hídricas das fontes de abastecimento e a capacidade das mesmas atenderem a população dentro de uma visão sustentável e de longo prazo.

$\mathrm{Na}$ realidade brasileira e de muitos países em desenvolvimento, se observa que as cidades de montante que não tratam esgoto contaminam as fontes das cidades de jusante, de forma que as cidades mais a jusante praticamente utilizam esgoto sem tratamento misturado com a vazão incremental, que também recebe outras contaminaçóes. Isto resulta num risco importante para a populaçáo, devido a insegurança dos mananciais. Além disso, existem cenários na própria cidade que áreas de mananciais contaminam as fontes de abastecimento. $\mathrm{O}$ resultado deste processo é a perda de mananciais e aumento do risco de saúde para a população. A seca de 2014-2015 no Sudeste do Brasil mostrou, que não houve falta de água, mas de água não contaminada, afinal o rio Tietê e Pinheiros em Sáo Paulo não secaram, mas estavam contaminados. A seca se torna mais grave quando a vazão natural diminui, perdendo a sua capacidade diluidora.

\section{Indicadores}

Para avaliar este processo Tucci (2017) apresentou dois indicadores:

- Vazão subsídio que é a vazão necessária para diluir o esgoto que não foi tratado. Somando este valor ao valor consumido, obtém-se que a vazão de subsídio chega a valores maiores que 200 vezes a vazão consumida para grande parte das cidades brasileiras. Este indicador retrata a deficiência do tratamento de esgoto na realidade do país. Esta ineficiência esta cobrando um preço muito alto para sociedade na insegurança da saúde e no impacto ambiental;

- Indicador de Sustentabilidade Hidrica - relaciona a demanda pela disponibilidade hídrica. Este indicador mostra que numa área urbana são necessários várias vezes a sua área em recursos naturais para atender a demanda devido a falta de tratamento de esgoto.
Este artigo apresenta os resultados destes indicadores para o Estado de Santa Catarina, analisando a variabilidade destes indicadores para todas as cidades no cenário atual e para uma proposta de Plano de Investimento em esgoto para o Estado, desenvolvido em três fases.

\section{Diagnóstico}

A demanda total em Santa Catarina é de $556 \mathrm{~m}^{3} / \mathrm{s}$ $\left(8,53 \mathrm{~m}^{3} / \mathrm{dia} / \mathrm{hab}\right.$. $)$ onde a vazão de subsídio representa $99,6 \%$ da vazão total. No Brasil, a demanda total per capita é de 7,53 m³/hab./dia. Assim, constata-se que a demanda total per capita obtida para as cidades de Santa Catarina é $13 \%$ superior ao verificado para o Brasil como um todo.

Considerando a $\mathrm{Q}_{95}$ como indicador de disponibilidade hídrica, os valores do IS $_{h}$ variaram entre 1,87 (em rancho Queimado - melhor desempenho) e 234,1 (em Mondaí - pior desempenho), com valor global para o Estado de 44,12. Isto indica que no Estado os municípios usam 44 vezes a sua área de ocupação para atender a demanda real, principalmente devido a falta de tratamento de esgoto. Quando isto não ocorre os rios ficam totalmente poluídos. Afinal, você conhece algum rio urbano deste país que não está poluído? Isto mostra o fracasso do setor de saneamento.

\section{Plano de Investimento}

Foram consideradas três fases quanto a melhoria da coleta e tratamento de esgoto, que são as seguintes:

Fase A - 95\% da população urbana atendida com coleta e tratamento de esgoto com uma eficiência de remoção da carga poluente de $80 \%$. Os investimentos num horizonte de 15 anos;

Fase B - A meta final 98\% da população urbana com acesso a coleta de e tratamento de esgoto e $80 \%$ de eficiência na remoção da carga poluente. $\mathrm{O}$ horizonte de tempo considerado para atingir a meta foi de 5 anos, após a fase A;

Fase C - Meta final 100\% de esgoto coletado e tratado nas 12 cidades de Santa Catarina com mais de 100 mil habitantes e eficiência de $95 \%$ na remoção da carga poluente nestes municípios. O horizonte de tempo considerado para atingir a meta também foi de 5 anos, após a fase $B$. 
Os resultados mostram o seguinte:

- Ao final da fase A espera-se uma redução de 66\% na vazão de subsídio per capita em relação ao cenário atual. Com a implementação da fase $\mathrm{B}$, espera-se uma reduçáo adicional de $3 \%$ em relação cenário atual, atingindo $70 \%$ de redução global. Por fim, ao final da fase $\mathrm{C}$ espera-se uma redução incremental de $11 \%$, atingindo $81 \%$ de redução da vazão de subsídio per capita com relação ao diagnóstico;

- Com relação a evolução do índice de sustentabilidade hídrica, no final da fase C haverá uma redução total de $73 \%$ com relação no cenário atual, indo de 44 para 12 . Isto significa que as cidades ainda terấo um uso de 12 vezes a sua área ocupada;

- O investimento na fase A é de R \$ 5,6 bilhóes e representa 2,23\% do PIB atual do Estado. Escalonados em 15 anos, o investimento anual da fase A é de $\mathrm{R} \$ 371$ milhóes, representando $0,12 \%$ do PIB a cada ano. O investimento estimado para a Fase B é R \$ 313 milhóes, 0,13\% do PIB. O investimento estimado para a fase C é R\$ 602 milhôes, 0,24\% do PIB estadual.

Este Plano de Investimento leva o Estado a um novo patamar ambiental dos rios e com redução dos riscos de saúde. Estas fases de investimentos representam a busca de um patamar de serviço que reduz grande parte das cargas. A evolução após este Plano de Investimento poderá envolver a busca de eficiência e conservação da água, além de solução de problemas específicos dentro de metas específicas de enquadramento de cada rio. A eficiência envolve o uso de tecnologia para reduzir o uso da água como contratos de "performance" para dedução de perdas. A conservação é o aumento do uso racional da água pela população. $\mathrm{O}$ uso de regularização de vazão não foi explorado neste artigo porque isto significaria construir barragens para diluir esgoto, o que é totalmente contrário aos princípios de sustentabilidade hídrica e ambiental.

\section{AGRADECIMENTOS}

Parte deste estudo foi realizada no âmbito do Projeto "Inovação em instrumentos da política ambiental em Santa Catarina - Recursos Hídricos", financiado pela Fundação e Amparo à Pesquisa e Inovação do Estado de Santa Catarina (FAPESC), o qual entre outras atividades foi responsável pela elaboração do Plano Estadual de Recursos Hídricos (PERH/SC), desenvolvido pelo Centro de Economia Verde (CEV) da Fundação CERTI para a Diretoria de Recursos Hídricos (DRHI) da Secretaria de Estado do Desenvolvimento Econômico Sustentável (SDS).

\section{REFERÊNCIAS}

AGÊNCIA NACIONAL DE ÁGUAS - ANA. Secretaria Nacional de Saneamento Ambiental. Atlas esgotos: despoluição de bacias hidrográficas. Brasília: ANA, 2017. Disponível em: <http://atlasesgotos. ana.gov.br/>. Acesso em: 2 abr. 2019.

BRASIL. Conselho Nacional do Meio Ambiente - CONAMA. Resolução n 357, de 17 de março de 2005. Diário Oficial [da] República Federativa do Brasil, Poder Executivo, Brasília, DF, 18 mar. 2015.

FEKETE, B.; STAKHIV, E. Performance indicators in the water resources management sector. In: BHARAURI, A. et al. (Orgs). The global water system in the anthropocene. Springer Water, 2014. chap. 2.

GEOAMBIENTE SENSORIAMENTO REMOTO LTDA. Projeto de proteção da mata atlântica em Santa Catarina (PPMA/SC). São José dos Campos, 2008. 90 p. Relatório Técnico do Mapeamento Temático Geral do Estado de Santa Catarina.

HOOPER, B. Key performance Indicators of River Basin organizations: visiting Scholar Program US Army Corps of Engineers. 2006. 100 p.

INSTITUTO BRASILEIRO DE GEOGRAFIA E ESTATÍSTICA - IBGE. Censo 2010. Rio de Janeiro, 2010. Disponível em: <http://censo2010. ibge.gov.br/>. Acesso em: 2 abr. 2019.

INSTITUTO BRASILEIRO DE GEOGRAFIA E ESTATÍSTICA - IBGE. Panorama geral de Santa Catarina. Rio de Janeiro, 2017. Disponível em: <https://cidades.ibge.gov.br/brasil/sc/panorama/>. Acesso em: 2 abr. 2019.

PAHL-WOSTL, C. A conceptual framework for analyzing adaptive capacity and multi-level learning processes in resource governance regimes. Global Environmental Change, Guildford, v. 19, p. 354-365, 2009. http://dx.doi.org/10.1016/j.gloenvcha.2009.06.001.

PANDOLFO, C. et al. Atlas climatológico do Estado de Santa Catarina. Florianópolis: Epagri, 2002. CD-ROM.

SANTACATARINA. Secretaria de Estado da Agricultura e Desenvolvimento Rural de Santa Catarina - SADR. Estudos dos instrumentos de gestão de recursos hídricos para o Estado de Santa Catarina e apoio a sua 
implementação: regionalização de vazões das bacias hidrográficas do Estado de Santa Catarina. Florianópolis: SADR, SDS, EngecorpsTetraplan-Lacaz Martins, 2006. 141 p.

SISTEMANACIONAL DE INFORMAÇÕES SOBRE SANEAMENTO SNIS. Série histórica de informações municipais. 2015. Disponível em: <http://app.cidades.gov.br/serieHistorica/>. Acesso em: 2 abr. 2019.
TUCCI, C. E. M. Integrated urban water management in large cities. World Bank, 2009. 166 p.

TUCCI, C. E. M. Indicador de sustentabilidade hídrica urbana. Revista de Gestão de Água da América Latina, Porto Alegre, v. 14, p. e7, 2017.

VON SPERLING, M. Introdução à qualidade das águas e ao tratamento de esgotos. 3. ed. Belo Horizonte: DESA, UFMG, 2005. 240 p.
Marcelo Pedroso Curtarelli

Contribuição do autor:

Carlos Eduardo M. Tucci

Contribuição do autor:

Vinicius Ragghianti

Contribuição do autor:

\section{Vitor Guimarães}

Contribuição do autor:
Certi.mpedroso.curtarelli@gmail.com

Contribuiu com a concepção do estudo, escrita do manuscrito, coleta e análise de dados, análise dos resultados e conclusóes.

Rhama.carlos.tucci@rhama.com.br

Contribuiu com a concepção do estudo, desenvolvimento da metodologia, construção dos cenários de investimento, análise dos resultados e conclusôes.

Certi.vtr@certi.org.br

Contribuiu com a concepção do estudo, desenvolvimento da metodologia, análise dos cenários de investimentos e conclusóes.

Certi.vsg@certi.org.br

Contribuiu com a coleta e análise de dados e análise dos resultados. 